\title{
Quaderni
}

QUADERNI Communication, technologies, pouvoir

\section{Les tendances innovatrices des sociétés contemporaines}

Innovative trends in contemporary societies

\section{Bernard Paulré}

\section{(2) OpenEdition \\ 1 Journals}

\section{Édition électronique}

URL : http://journals.openedition.org/quaderni/975

DOI : 10.4000/quaderni.975

ISSN : 2105-2956

Éditeur

Les éditions de la Maison des sciences de l'Homme

\section{Édition imprimée}

Date de publication : 5 mai 2016

Pagination : 5-20

\section{Référence électronique}

Bernard Paulré, «Les tendances innovatrices des sociétés contemporaines », Quaderni [En ligne], 90 | Printemps 2016, mis en ligne le 05 mai 2016, consulté le 23 septembre 2020. URL : http://

journals.openedition.org/quaderni/975; DOI : https://doi.org/10.4000/quaderni.975 


\section{$D$ os sier}

\section{Les tendances innovatrices des sociétés contemporaines}

Bernard Paulré

Université Paris 1 Panthéon Sorbonne

\section{Position des problèmes}

L'innovation fut introduite dans la théorie économique par J. Schumpeter en $1911^{1}$. Cette notion appartient désormais à un fonds d'analyse commun exploité aussi bien par les économistes orthodoxes qu'hétérodoxes. Si les approches se sont fortement différenciées avec le temps, et si les représentations se sont parfois complexifiées ${ }^{2}$, tout économiste sait ou pense savoir de quoi il est question quand on utilise le mot innovation. Pour l'essentiel :

- une nouveauté techno-économique constituant le plus souvent une rupture par rapport aux objets, ressources et/ou procédés existants,

- un acteur ou un groupe d'acteurs à l'origine de l'innovation : un entrepreneur travaillant à l'inspiration et à la conviction, exploitant une invention ou les résultats d'une activité de R\&D ; une entreprise (englobant éventuellement un département de $R \& D$ ) ou encore un réseau d'acteurs...

- une sanction et une évaluation de l'innovation en son contenu (un produit, un service nouveau ou un procédé nouveau...), effectuées essentiellement via le marché,

- un investissement auquel est associée une rentabilité (protégée, le plus souvent, par un système de droits) ce qui implique une rationalité économique,

- une activité qui se déploie pour sa majeure partie, de bout en bout, au sein du système économique tout en y jouant un rôle perturbateur. 
Nous reviendrons plus longuement sur l'approche économique (cf. article de B. Paulré dans ce numéro). Car si nous introduisons notre propos en évoquant, à grands traits, cette représentation de l'innovation, c'est pour introduire deux observations complémentaires. La première est que, tout au long du $\mathrm{XX}^{\mathrm{e}}$ siècle au moins, l'innovation technologique a été associée étroitement, d'une part, au capitalisme industriel et à l'économie de marché et, d'autre part, à l'emprise quasi-idéologique de l'objectif de croissance industrielle, de la recherche de gains de productivité et, plus globalement, de la volonté de modernisation. C'est ce type d'innovation qui a monopolisé l'attention au point que le mot, utilisé sans adjectif relationnel, connotait à peu près inévitablement l'innovation techno-économique. Certes, à la même période, le mot innovation a aussi été utilisé, en sociologie notamment, dans son sens originel d'introduction d'une nouveauté ${ }^{3}$. Mais, pendant longtemps, cela n'a pu, au mieux, que contribuer à nuancer, selon nous, la primauté sémantique implicite donnée à la production et à la technique.

La seconde raison est qu'aujourd'hui, soit par une prise de distance assumée avec l'économie industrielle, soit, ce que nous sommes enclins à privilégier, à la suite d'une évolution historique non achevée et dont le sens est à identifier, nous sommes confrontés à une situation nouvelle et originale caractérisable, selon nous, par trois traits :

1. Le mot innovation est de plus en plus utilisé en dehors du champ économique pour désigner des activités que l'on peut qualifier, génériquement, de «créatrices de nouveauté» et dont les modalités et les contenus sont sensiblement différents de ceux de l'innovation économique : innovation financière $^{4}$, innovation sociale ${ }^{5}$, innovation en éducation ${ }^{6}$, innovation territoriale ${ }^{7}$, innovation institutionnelle ${ }^{8}$, innovation politique ${ }^{9}$, innovation juridique $e^{10}$ et même innovation théologique $^{11}$.

La liste des domaines dans lesquels on innove, ou dans lesquels on prétend innover, semble aujourd'hui presque sans limite. La société semble avoir été envahie dans sa totalité par la poursuite de l'innovation. «Ça» parle beaucoup d'innovation dans les sociétés contemporaines et la question se pose donc de savoir de quoi on parle et pourquoi on en parle tant. Allons plus loin : ne serait-on pas davantage dans l'ordre du discours et de la rhétorique ? La réalité de la nouveauté en jeu ne serait-elle pas parfois secondaire voire de l'ordre du performatif? Mais à supposer que cette prégnance d'un discours de soutien, de promotion ou d'institutionnalisation de l'innovation soit effective, demeure encore la question de savoir pourquoi elle occupe une place importante dans les discours, et notamment, ce qui est relativement nouveau, dans les discours de légitimation de certaines actions publiques ${ }^{12}$.

Nous ne pouvons, dans le cadre de cette publication, recenser et illustrer les diverses manifestations de l'innovation ne relevant pas de l'innovation économique au sens habituel. L'intérêt en serait évidemment indéniable pour en faire ressortir les significations concrètes spécifiques et leur variété. Nous avons préféré illustrer notre propos, d'abord, par l'approfondissement d'un domaine : celui de l'innovation sociale qui est peut-être le domaine « non économique » où la notion d'innovation s'est le plus répandue ${ }^{13}$. 
On peut définir les innovations sociales comme " de nouvelles idées (produits, services et modèles) qui, simultanément, répondent à des besoins sociaux... et qui créent de nouvelles relations ou collaborations sociales $\gg^{14}$. Ces innovations sont donc sociales à la fois dans leurs fins et dans leurs moyens. Mais la nouveauté semble se situer ici du côté de l'organisation et des relations sociales grâce auxquelles certains besoins sociaux vont être satisfaits si bien que, selon nous, le modèle de l'innovation sociale est un modèle rationaliste et de négociation sociopolitique. Ce que souligne bien P. Leduc Browne dans sa contribution à ce numéro : «L'innovation sociale... n'est plus définie comme une idée, une pratique ou une manière de faire qui répond à un besoin, mais plutôt comme l'innovation au sein des rapports sociaux, c'est-à-dire comme l'amorce de véritables transformations dans les rapports de pouvoir qui structurent les sociétés.»

P. Leduc Browne observe également que l'« innovation sociale » n'est devenue une notion courante et répandue que depuis les années 1990 même si, souligne-t-il, « on peut trouver le terme 'innovations sociales' chez $V$. Considérant ou 'invention sociale' chez M. Weber... ». Reprenant une remarque de B. Godin, il écrit aussi que « la montée récente de l'innovation sociale constituerait une réaction à l'hégémonie de l'innovation technologique. »

Même si ce n'est pas dans une logique d'inventaire, bon nombre d'autres secteurs ou domaines dans lesquels se manifeste aujourd'hui une volonté d'innovation seront abordés dans ce double numéro spécial. Ainsi, dès le présent numéro, la philosophie nous fournira une illus- tration particulièrement éclairante de cette volonté d'innover en dehors du champ économique. L'illustration est d'autant plus significative que l'on ne s'attend pas à trouver cette discipline comme exemple d'un lieu où se manifestent cette intention et une certaine valorisation de l'innovation. Nous y reviendrons ci-dessous à propos de la contribution à ce numéro de Charles Ramond.

Les autres domaines qui seront abordés sont : le secteur universitaire (Claude-Julie Bourque dans le présent numéro), le domaine institutionnel (Thierry Delpeuch dans le second numéro), le management de l'innovation et de la créativité (Emma Jeanes, dans le second numéro également). Dans le second numéro Danilo Martuccelli introduira le point de vue du sociologue, et l'on y traitera aussi de la façon dont le thème de l'innovation est abordé par quelques écrivains contemporains.

Un numéro spécial de la Revue de Métaphysique et de Morale a été consacré à l'innovation en $2014^{15}$. L'orientation générale est très différente du projet qui inspire ce double numéro de Quaderni mais on y relève au moins cette observation partagée : "la naissance de la nouveauté montre une variété de modalités ; des profils d'innovation différents " selon les domaines de compétences (p. 301).

2. On observe des injonctions à innover qui peuvent sembler paradoxales.

Les sociétés contemporaines ou, du moins, la plupart des acteurs qui ont des postes de responsabilité, publics comme privés, semblent comme obsédés par l'innovation, souvent comprise 
comme une condition de la survie (individuelle et collective). On exhorte chacun, quelles que soient ses activités, à innover ou à participer à des activités innovatrices. On peut cependant considérer que, dans la mesure où la volonté et la conviction d'avoir une idée originale peuvent être des facteurs importants de l'innovation ${ }^{16}$, l'injonction à innover a un côté paradoxal ${ }^{17}$ et peut avoir pour effet d'affaiblir l'engagement dans cette activité ou faire perdre au mot son sens originel.

Nous n'avons pas cherché à illustrer dans le détail l'injonction à innover et à en recenser les diverses manifestations ainsi que les acteurs impliqués. Il nous a paru plus intéressant d'examiner empiriquement comment ces injonctions se manifestent et quels en sont les effets, dont on devine qu'ils peuvent parfois (sinon souvent) être contreproductifs. Pour cela, nous avons choisi d'illustrer principalement ce phénomène dans l'enseignement supérieur et la recherche, secteur qui semble fortement impacté par les injonctions des responsables politiques. Tel est l'objet de la contribution de Claude-Julie Bourque dont la richesse des résultats qualitatifs rend difficile leur synthèse. Nous en retenons ici trois éléments de conclusion : 1) "Les chercheurs donnent à la notion d'innovation un sens axé sur son potentiel révolutionnaire plus proche de Schumpeter dans sa dimension socio-économique et de Kuhn dans sa dimension paradigmatique »; 2) Ils «ne s'opposent pas à ce que les gouvernements stimulent des innovations porteuses de retombées économiques, mais plutôt à ce que ce modèle s'impose à l'ensemble du champ scientifique », et 3) « [ils] mettent en cause les logiques politiques, managériales et comptables souvent incompatibles avec la production de connaissances qui reste pour eux l'objectif ultime qui distingue la recherche scientifique universitaire (fondamentale et appliquée) de la R-D (commerciale et industrielle)».

Deux raisons nous ont incité à solliciter ClaudeJulie Bourque : d'abord la volonté de ne pas privilégier les analyses centrées sur les sciences humaines, comme cela est souvent le cas. Non pas que le cas de ces disciplines n'est pas intéressant. Le problème est qu'elles soulèvent, par leur nature, des difficultés spécifiques qui accentuent ou singularisent la portée des discours et des injonctions à innover. Nous avons donc préféré mettre l'accent sur l'étude des réactions aux injonctions à innover dans le secteur des sciences dites « dures » (ici : les sciences de la vie et de la santé). Ensuite, nous avons choisi de nous adresser à un chercheur canadien ce qui permet de décentrer l'analyse, c'est-à-dire de prendre un peu de distance avec le cas français, et donc de montrer comment les effets de l'injonction se manifestent dans un autre pays. Mais à lire les résultats des enquêtes de C.-J. Bourque, nous n'imaginons pas que les enseignements généraux qu'elle en tire pour le Canada puissent être très différents de ceux que l'on pourrait retenir d'une enquête analogue menée en France, comme le suggère l'auteure, qui cite d'ailleurs une enquête dont les résultats ont été publiés dans Quaderni en $2012^{18}$.

\section{3. l'innovation est souvent l'objet d'une valori- sation en soi.}

Dans le schéma classique de l'économie, l'innovation est désirée pour ce qu'elle apporte : un produit, un service ou un procédé nouveau pour 
l'essentiel. Le marché sanctionne le résultat de l'innovation c'est-à-dire ce à travers quoi elle se manifeste finalement. Il contribue à sa diffusion. Or on assiste aujourd'hui à une double valorisation: de l'acte d'innover et de celui qui innove. Au point qu'à la limite, on peut se demander si l'innovation ne serait pas moins désirée pour ce qu'elle produit, que pour ce qu'elle est intrinsèquement comme action et ce qu'elle représente en terme de performance ou de reconnaissance d'une capacité individuelle ${ }^{19}$. Cette observation rejoint en partie la description psychologique de l'entrepreneur que fait Schumpeter en $1911^{20}$. En partie seulement parce que l'entrepreneur « schumpeterien » ne trouve pas sa satisfaction dans la reconnaissance des autres et dans la valorisation de ce qu'il est, mais dans l'acte même d'entreprendre. C'est pourquoi notre observation sur la valorisation contemporaine de l'innovation semble plutôt rejoindre le tableau dressé par A. Ehrenberg dans Le culte de la performance, qu'elle vient en quelque sorte compléter et renforcer.

Là encore, nous pourrions multiplier les illustrations de ce phénomène de la valorisation en soi de l'innovation comme acte. Le domaine qui permet ici d'illustrer précisément à quel point cette valorisation occupe une place importante, est la philosophie. Cette illustration est d'autant plus marquante et significative que l'on peut légitimement penser que cette discipline est normalement à l'écart de ce type de préoccupation. C'est tout l'intérêt de la contribution de Charles Ramond au présent numéro que de nous montrer, sur un cas d'autant plus intéressant qu'il concerne l'un des grands philosophes du $\mathrm{XX}^{\mathrm{e}}$ siècle, Michel Foucault, comment la nouveauté semble avoir changé de statut avec celui-ci : alors que jusqu'à lui, la valorisation de la nouveauté ne se posait pas, y compris et surtout pour les philosophes qui ont créé des systèmes philosophiques « nouveaux » (Descartes par exemple, mais aussi Nietzche, Bergson etc.), avec Foucault le changement de paradigme acquiert une valeur en soi, peut-être même indépendamment de la question de savoir quel en est au fond l'intérêt. C. Ramond nous fait en effet découvrir la position paradoxale de M. Foucault qui, sur la base d'une conception somme toute traditionnelle de la philosophie, semble avoir retardé, c'est-àdire différé, l'étude de l'apport de la nouveauté.

En conclusion, face à une situation nouvelle dont on ne peut, pour des raisons de place évidentes, que suggérer partiellement la réalité et le caractère original, le cadre d'interprétation et l'outillage empruntés à ou adaptés de l'économie sont obsolètes et nous devons y renoncer. Non seulement sur le plan conceptuel et de la représentation du phénomène, mais aussi et surtout sur le fond, c'est-à-dire pour la compréhension des innovations et des formes de changement des sociétés contemporaines qui leur sont associées. Nous allons prolonger notre approche du problème en suggérant quelques pistes de réflexion permettant de mettre en perspective ce qui nous semble constituer le nouveau paysage de l'innovation dans les sociétés contemporaines.

\section{Les pistes de réflexion}

Pour ordonner les pistes d'interprétation et de recherche nouvelles qu'appelle la nouvelle situation, nous allons distinguer la réflexion conceptuelle de celles relatives à la signification 
historique de l'évolution en cours.

1. Sur le plan conceptuel, la signification et le sens de l'usage du mot innovation sont aujourd'hui des questions ouvertes.

Selon nous, le problème de la signification du mot innovation est posé non seulement en dehors de l'économie, mais aussi en économie même où la représentation de l'activité innovante et de ses effets s'est sensiblement développée et approfondie depuis la fin $\mathrm{du} \mathrm{XX}^{\mathrm{e}}$ siècle au point de fournir des représentations qui n'ont plus rien à voir avec les visions schumpétériennes initiales (cf. article de B. Paulré). Si l'on peut opposer la conception schumpétérienne de l'innovation économique et les formes ou la signification contemporaines de l'innovation dans des domaines autres qu'économiques, on notera que la tension de cette opposition sera moins forte si l'on se réfère à des représentations très récentes de l'innovation en économie : par exemple, l'approche en termes de Système National d'Innovation qui repose sur une conception diffuse des activités innovatrices, encastrées dans une grande partie de la société. Mais, ce faisant, on se place davantage sur le terrain de la représentation du processus d'innovation que de la sémantique du mot, même s'il est difficile de considérer que ces deux aspects sont totalement indépendants. Revenons-en donc à la question sémantique stricte.

B. Godin est sans doute le chercheur qui a le plus étudié l'évolution et la genèse historique du sens du mot innovation, et dont l'apport à ce numéro est par conséquent le plus riche à ce propos. Il montre que, pendant une très longue période, l'innovation était un acte répréhensible ou contestable, et que le concept d'innovation a ainsi eu, pendant très longtemps, une signification essentiellement péjorative. Il documente ainsi une historiographie ( encore à faire ») du concept d'innovation et note que les réflexions actuelles sont « empreintes de mythes et de confusions conceptuelles ». Il montre comment et pourquoi « ce concept est devenu, et ce en moins d'un siècle, une valeur et un slogan ». Son article présente ainsi l'intérêt de mener de concert une approche historique sémantique et une étude de la valorisation c'est-à-dire de la valence sociale de l'innovation. Mais, soulignons-le, la valence en question concerne la valorisation sociale de l'innovation comme acte porteur d'une nouveauté et non, comme nous l'avons évoqué précédemment, comme acte valorisable en soi c'est-àdire comme performance.

Cette réflexion conceptuelle très stimulante peut être prolongée et nous permet d'envisager à propos de l'innovation une orientation de recherche qui rompt avec le positivisme auquel la plupart des économistes (mais ils ne sont pas les seuls) sont enclins, et qui les amène à raisonner à partir d'un concept destiné à rendre compte d'une certaine « réalité ». Cette orientation est intéressante en ce qu'elle permet aussi de faire le lien avec la valorisation, aussi bien en ce qui concerne le contenu de l'innovation que l'acte en soi.

L'idée est de promouvoir un approfondissement de la recherche sémantique sur l'usage et le sens du mot innovation en l'élargissant, c'est-à-dire en allant au-delà du recensement de ses usages passés et en introduisant une dimension sociale. Il s'agit d'insister sur le caractère « endogène » de la qualification de l'innovation, soutenus par ce que nous observons, à savoir une prise en 
charge langagière délibérée et tâtonnante de la notion d'innovation dans des domaines d'où elle semblait absente sinon exclue (ou l'on parlait à la rigueur de changement...). Non seulement l'innovation a envahi la société mais, de plus, dans le même mouvement, les acteurs font évoluer et travaillent le langage donc la "réalité » ou la nature du phénomène. Ce qui ne doit pas surprendre car avec la multiplication de ses usages et de ses contextes, on comprend bien que le sens du mot innovation devient l'objet d'un effort de transposition ou d'adaptation.

On ne peut contester la nécessité d'étudier ce travail langagier dès lors que l'on accepte de considérer que l'approche du " réel » passe nécessairement par le langage. Étudier l'innovation c'est ainsi à la fois étudier des faits, une «réalité», et étudier la façon dont ils sont appréhendés au niveau de la langue. Or, en posant le principe d'un rapport exclusif entre une chose et sa dénomination, comme cela se pratique empiriquement souvent, on affirme l'unité, sinon l'universalité d'un phénomène, alors même que la variété et les spécificités de ses manifestations actuelles constituent autant d'obstacles à son identification. C'est bien pour cette raison que la sémantique du mot innovation est travaillée, implicitement, via ses multiples usages.

Prendre ainsi un « tournant linguistique $»^{21}$, c'est partir du principe que l'innovation n'est pas un fait mais plutôt, et d'abord, une interprétation ou une représentation ${ }^{22}$. Elle peut être considérée comme un fait de société pour la raison qu'elle résulte, pour une part importante, de la vision que la société (ou un groupe, ou encore un secteur particulier) tend à donner d'elle-même, et que se développent à son sujet des discours variés. L'article de P. Leduc Browne est illustratif de notre propos car il montre bien que l'innovation sociale est une «notion qui se cherche ». Mais ce qui est en jeu, selon nous, ce n'est pas simplement la définition d'un concept nouveau. C'est surtout l'invariant réflexif d'une famille de pratiques mettant en relation des acteurs produisant des relations nouvelles et qui se reconnaissent dans une expression sémantique. D'une autre façon, C.-J. Bourque illustre aussi, dans une section baptisée « le sens des mots » une certaine volatilité sémantique qui s'accorde bien, selon nous, au fait que les chercheurs ne s'approprient pas toujours le concept tel qu'il semble entendu par ailleurs. Dans un autre ordre d'idées, lorsque L. Sfez ${ }^{23}$ forge le néologisme d'innovention pour exprimer le lien entre la science fondatrice qui invente et la technique servante qui innove, il souligne la nouveauté d'une situation qui ne se laisse pas saisir par le vocabulaire traditionnel.

Nous pouvons ici faire le lien entre notre propos et l'histoire des concepts selon R. Koselleck ${ }^{24}$. D'abord pour poser le problème de la conceptualisation de l'innovation. Ensuite pour souligner la pertinence de l'étude de la relation entre le sens du mot et les contextes sociaux dans lesquels on l'utilise aujourd'hui.

Selon B. Müller, pour Koselleck, « une histoire conceptuelle n'a pas pour unique objet l'interprétation des variations sémantiques d'entités intellectuelles ä partir de leur seule expression linguistique. Les concepts sont aussi l'expression des luttes sociales, politiques ou intellectuelles; ils sont eux-mêmes enjeu de ces luttes et, au travers des expressions sémantiques, ce sont 
des états particuliers de ces luttes qui sont ainsi fixés lexicalement et dont l'historien peut rendre compte $»^{25}$.

Ce faisant, nous ne nous inscrivons pas dans le cadre d'une historiographie car c'est aux usages actuels du mot innovation que nous nous intéressons. En partant du principe qu'une mutation est en cours et que la grille linguistique traditionnelle est obsolète, c'est vers une «histoire du temps présent» que nous nous tournons. La question posée n'est donc pas seulement: Qu'est-ce qu'innover aujourd'hui ? Elle est aussi de savoir quelle est la signification sociale (et aussi institutionnelle et politique le cas échéant) de l'usage du mot, et qui l'utilise. Il doit s'agir ainsi, par exemple, de s'interroger sur cet usage comme légitimation ou comme labellisation d'un certain nombre d'activités engagées dans l'administration. Au travers de tous les usages que l'on recense, dont certains relèvent de la sphère institutionnelle et politique, il y a manifestement des enjeux. Dont certains sont symboliques, mais pas uniquement.

2. Sur le fond, la question posée est celle de savoir quelle est la signification historique de la situation caractérisée ci-dessus. Que peut-on dire de l'évolution des sociétés contemporaines à partir de cette observation?

a) Dans une première direction, d'inspiration en partie économique, on peut voir dans cette évolution et dans les phénomènes observés la manifestation d'une emprise forte du néo-libéralisme sur les sociétés développées contemporaines.

Selon Foucault, le néolibéralisme peut être conçu comme un mode de gouvernementalité. Le développement de cette ligne de pensée conduit à considérer l'individu comme un entrepreneur de soi (interprétation de la théorie du capital humain par Foucault). La croyance au pouvoir régulateur du marché, jointe à la thèse d'une gouvernementalité axée sur les capacités et la rationalité de l'individu, cela dans un contexte de globalisation qui accentue la nécessité de l'adaptation, tant micro que macroéconomique, peut justifier l'appel à l'innovation partout où cela est possible, tant sur les marchés stricto sensu que dans la société en général.

L'innovation est en effet une forme de concurrence alternative à la concurrence par les prix. L'injonction à innover est donc une autre forme d'injonction visant à faire exercer par chacun son pouvoir concurrentiel, non pas en jouant sur les coûts ou sur la saisie des opportunités de marché, mais en jouant sur la capacité à perturber le système à son propre avantage par l'innovation.

Pourquoi dans le domaine social, c'est-à-dire en dehors du marché ? Si l'on suit Foucault, une forme de gouvernement à distance remplace le gouvernement direct par l'État. Dans cette perspective, des dispositifs ou des arrangements sociaux nouveaux sont nécessaires pour responsabiliser de différentes façons les acteurs. Les injonctions à innover et les processus originaux de négociation ou d'institutionnalisation, notamment dans le domaine social et politique, peuvent créer une responsabilisation et des formes de rationalité qui manifesteront l'emprise étendue du marché dans la société. Mais elles s'expliquent aussi par la nécessité d'intervenir au-delà du marché lui-même compte tenu de ses limites intrinsèques (externalités, bien collectifs, incer- 
titude...) tout en orientant les acteurs vers des conduites concurrentielles et fondées en partie sur une rationalité économique. Autrement dit, il s'agit de conduire les individus à se réaliser comme entrepreneur (de type schumpétérien) et à intérioriser les contraintes et les modes d'évaluation publics auxquels ils sont exposés, de sorte que leur action s'oriente « spontanément 》 vers la recherche de l'efficacité et l'acceptation de ces évaluations.

b) Dans une deuxième direction, on peut faire le lien avec les thèses sur ce que, génériquement, nous appelons l'après-modernité.

La nature de l'innovation est ambiguë. D'un côté, par son origine, elle est un marqueur de la modernité. Mais de l'autre, par la diversité actuelle de ses manifestations, par son caractère permanent, son accélération et certains excès, on peut la considérer comme l'une des manifestations des dérives de cette modernité.

Marqueur de la modernité, l'innovation l'est certainement dans la mesure où elle manifeste l'intention de tourner le dos à la routine et reflète la volonté d'introduire du changement dans la sphère économique. C'est ce que Schumpeter exprime clairement en 1911, en opposant ce qu'il appelle le « circuit» (monde de l'économie répétitive et stationnaire) à l'évolution (période au cours de laquelle une innovation est introduite puis assimilée).

La modernité associe émancipation de l'humanité, rationalisation et progrès technique. Elle se manifeste au travers de l'évolution technique et industrielle, et promeut une conception de l'his- toire dans laquelle les hommes ont la volonté de transformer leur monde et le pouvoir d'être les acteurs de leur devenir. On est dans l'ordre du faire et de la logique instrumentale. La modernité est associée à une « religion du progrès » entendu comme principalement scientifique et technique.

On peut constater à quel point l'innovation, dans sa conception schumpétérienne au moins, participe de la modernité. Elle place au centre de l'analyse économique la question de la modernisation et du progrès technique d'origine industrielle. Et elle impute la responsabilité de ce progrès à un ou des individus (l'entrepreneur puis l'équipe de R\&D) mus par la volonté de changer et de contribuer au progrès et à la modernisation, leur reconnaissant la capacité à créer du changement et à repousser certaines limites techniques et/ou économiques. Enfin, elle identifie les facteurs qui jouent un rôle dans cette capacité ${ }^{6}$.

L'endogénéisation économique du progrès technique par Schumpeter fait aussi ressortir sa subordination à la demande et au marché. On ne parle d'innovation que pour désigner des combinaisons nouvelles ayant rencontré le succès commercial. Le marché opère une sélection. L'entrepreneur ou le grand groupe n'investissent que dans la mesure où ils ont la conviction et/ou la capacité de faire que l'offre rencontre une demande.

Associée aux dérives de la modernité, l'innovation l'est aujourd'hui aussi dans la mesure où : (1) elle change de nature et est présente partout de façon très diverse, (2) elle procède d'une dynamique et d'une accélération qui créent de l'éphémérité et des effets de mode, (3) elle s'inscrit dans une double dissociation : du social et de 
l'économie d'un côté, de ces deux univers et de la technique de l'autre.

Avec le recul, nous savons ce qu'il advint de l'innovation conçue comme marqueur de la modernité. À un certain moment la modernisation scientifique et technique se désolidarise des valeurs d'émancipation et de raison ${ }^{27}$. On peut aller jusqu'à considérer qu'elle devient à ellemême sa propre fin et que le système technique s'autonomise ${ }^{28}$.

On ne peut plus voir dans l'innovation la simple manifestation d'une volonté de modernisation et de progrès. Pour une part importante, elle semble procéder essentiellement du souci de créer au bénéfice des entreprises des occasions de profit ou l'opportunité d'exercer un pouvoir économique; au besoin en suscitant une demande pour des biens et services qui ne constituent pas de véritables facteurs de progrès. Les pseudo innovations pullulent. La mode scande l'évolution de la consommation et d'une partie importante de la sphère de la production. On s'éloigne d'une volonté de progrès pour aller dans le sens de la satisfaction primordiale d'objectifs commerciaux ou financiers privés. Manipulée par la publicité, la demande n'est plus le juge de touche indépendant. On ne parle plus de besoins mais de désirs. Et l'obsolescence tend à devenir de plus en plus programmée.

L'innovation économique obéit d'autant moins à une volonté de progrès qu'elle semble nécessaire aux grandes entreprises et aux nations pour affronter la concurrence internationale. La mondialisation tend à placer l'innovation au centre du jeu et génère cette nécessité impérieuse nouvelle sans laquelle la survie économique des pays développés et de leurs grandes entreprises serait menacée. Mais, nourrissant potentiellement aussi bien un techno-nationalisme qu' un technoglobalisme ${ }^{29}$, la portée et le sens de cet impératif demeurent ambigus.

Plus largement, la rationalité de cette dynamique innovatrice permanente nourrit elle-même un autre débat. Celui opposant un grand récit des changements technologiques et de consommation majeurs (la théorie schumpetérienne des cycles Kondratiev, ou celle des paradigmes technoéconomiques de C. Freeman et C. Perez) à des conceptions nouvelles et locales de l'innovation dont certaines sont « hors marché » (les innovations sociales par exemple). L'innovation n'est plus réservée au domaine techno-économique. Elle se généralise et change de nature : elle devient financière, sociale, organisationnelle, éducative... Et même techno-économique, l'innovation est souvent incrémentale, imitatrice et dissociée des cycles.

Enfin, de conviction et d'engagement l'innovation devient impératif et objet d'injonctions. D'objet de décision et d'occasion d'aventure et de rupture, elle risque de devenir elle-même routine ou programme, sinon automatisme. D'acte libre, autonome, créatif et inspiré, engagé par les acteurs du changement et du progrès dans un système capitaliste, l'innovation devient un impératif économique et de management.

Ce n'est plus le progrès en tant que tel qui légitime l'activité nouvelle, mais la capacité à créer (ou à prétendre créer) du changement au profit d'une entreprise ou d'une nation, à générer de 
la croissance ou encore à créer et façonner une demande. Le maintien d'un discours assimilant innovation et progrès peut s'expliquer par la volonté de légitimer un ordre capitaliste, ne serait-ce que dans la mesure où l'innovation demeure un marqueur du capitalisme.

On ne peut ouvrir ici un débat sur les différentes visions de l'après-modernité, ni sur le point de savoir si les tendances innovatrices des sociétés contemporaines vont dans le sens d'une post ou d'une hyper-modernité ${ }^{30}$. La situation nous semble d'ailleurs plus complexe. Nous nous contenterons de suggérer que, par l'accent et l'insistance mise sur l'imperium de la nouveauté et sur la réflexivité associée, l'enjeu, au moins, est clairement défini : l'innovation sera-t-elle un vecteur de libération, ou bien ne sera-t-elle qu'une nouvelle occasion d'asservissement, tant du côté de la production que de celui de la consommation?

c) Troisième piste de recherche : l'émergence d'une société d'innovation?

L'innovation pénètre la société de diverses façons. D'un côté l'analyse des processus d'innovation en économie repose, selon le courant évolutionniste, sur l'existence de systèmes nationaux ou locaux d'innovation, c'est-à-dire sur un encastrement complexe des opérations multiples qui contribuent à l'innovation, localisées dans des parties différentes de la société, bien au-delà des frontières des entreprises et des milieux industriels. De l'autre, nous y avons insisté, l'esprit et la volonté d'innovation non technoéconomique se manifestent dans bon nombre de secteurs variés. Au bout du compte, toute la société semble imprégnée et mobilisée (ou mobilisable) par l'esprit d'innovation et marquée par les temporalités et les rythmes de ce type d'activité. De phénomène en partie erratique et exceptionnel, à l'origine des cycles économiques, l'innovation est devenue une activité permanente, recherchée, repérée statistiquement, encadrée en partie et soutenue par des incitations. L'innovation est devenue une nouvelle « ardente obligation ». Certains diront même un slogan ${ }^{31}$.

L'activité et la volonté d'innovation se sont tellement diffusées qu'il n'est pas incongru de parler d'une société d'innovation ${ }^{32}$. Mais pas au sens où $\mathrm{P}$. Thibaud, le premier, introduisit cette notion en $1985^{33}$. Car il privilégiait encore, à l'époque, les innovations économiques et la production. Nous utilisons cette expression pour exprimer le fait que les sociétés contemporaines sont imprégnées d'une volonté d'innovation et traversées par un esprit d'innovation qui gagne quasiment tous les domaines d'activité et se manifeste sous des formes et selon des rationalités très variées. L'esprit d'innovation ne peut être réduit ou confondu avec l'esprit d'inventivité sur lequel insistait également Paul Thibaud. Innover, c'est faire entrer une nouveauté dans le réel (ou le prétendre) c'est-à-dire modifier concrètement la façon de faire ou les buts pratiques que l'on se fixe. Et c'est initier, ce faisant, un processus de diffusion ou de répétition de cette nouveauté, donc c'est inaugurer une trajectoire. Une innovation modifie le champ des pratiques possibles non à partir d'une idée, mais à partir d'une première réalisation, que l'on appellera prototype, expérimentation pilote ou version 0 , peu importe. L'étude de l'innovation ne se réduit pas non plus à celle du changement. Car l'innovation est une 
activité volontaire orientée par des buts et des objectifs, qui mobilise une personne ou un groupe de personnes ayant éventuellement, tournées vers un même objectif, des fonctions différentes, et qui visent à créer une forme d'activité nouvelle ou un produit nouveau.

Le concept de société d'innovation n'est pas éloigné de celui de capitalisme cognitif ${ }^{34}$. Ce qui n'est guère étonnant car, ouvrant le champ des possibles et exploitant des expériences, l'innovation repose sur la mobilisation et la production de connaissances. Quant au lien entre innovation et capitalisme nous avons vu qu'il est essentiel, au moins dans l'approche schumpétérienne. Préférant éviter ici de donner à cette présentation une connotation trop économique, c'est un lien que nous notons en passant.

Nous reviendrons sur d'autres mises en perspectives dans le second numéro de cette double livraison.

Retenons pour l'heure que, si l'on accepte la caractérisation de la façon dont se présente l'innovation dans les sociétés contemporaines, les trois mises en perspective esquissées ne sont pas incompatibles, dessinant peut être les contours du régime néo-libéral d'une société d'innovation dont la dynamique complexe serait l'une des manifestations de l'après-modernité c'est-à-dire, pour les uns, d'une modernité tardive et, pour les autres, d'une post-modernité.

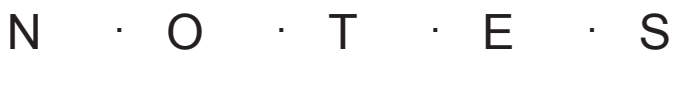

1. J. Schumpeter, Theorie der wirtschaftlichen Entwicklung, première édition, 1911. Traduction française : Théorie de l'évolution économique, Dalloz, 1935. Le mot innovation n'est pas utilisé dans le texte de 1911. J. Schumpeter ne l'utilisera qu'en 1928. Cf. à ce sujet les articles de B. Paulré et de B. Godin dans ce numéro.

2. L'épisode marquant le plus récent a été le développement de l'approche évolutionniste, plutôt hétérodoxe, au début des années 1980 (cf. B. Paulré, « L'apport de l'évolutionnisme contemporain à l'analyse économique de l'innovation », in Économie appliquée, 1997, n 3). Au même moment on a assisté au développement des théories de la croissance endogène, plutôt orthodoxes, qui placent l'innovation et les «idées» au cœur d'une croissance fondée sur des rendements croissants.

3. Nous pensons à l'innovation comme changement des règles du jeu (R. Boudon, La place du désordre, PUF, 1984), ou encore comme décision non programmée

(J. G. March et H. A. Simon, Les organisations, Dunod, trad. franç., 1964). Mais même dans un ouvrage très connu qui traite de la diffusion de l'innovation en général, et inspiré par les travaux du sociologue G. Tarde, on peut relever un net centrage sur la technique au point d'utiliser parfois de façon synonyme les mots innovation et nouvelle technologie : E. Rogers, Diffusion of innovations, $1^{\text {ère }}$ éd. : 1962 (The Free Press).

4. L'innovation financière ne s'analyse pas comme l'innovation technoéconomique. Ainsi, l'un des schémas bien connus des économistes pour expliquer les innovations financières est celui des stratégies de contournement (c'est-à-dire la volonté d'optimiser ou de contourner des règles prudentielles et comptables, 
selon la thèse de Silber W., 1983, "The Process of Financial Innovation", in American Economic Review, vol. $73, n^{\circ}$ 2). D'autre part, selon H. Mynski, élève de Schumpeter, les innovations financières contribuent à l'instabilité des systèmes financiers qui serait une caractéristique des économies capitalistes modernes. Concernant la crise des subprimes, il semblerait que ce soit le rythme accéléré de l'innovation financière et la complexité des produits financiers proposés par les banques qui, surclassant la capacité des acteurs financiers à appréhender les risques, aient provoqué un certain nombre de disfonctionnements. Bref, les innovations financières procèdent de comportements spécifiques et se propagent selon des voies différentes de celles de l'innovation industrielle. Cf. notamment : H. Minsky, 1982, “The financial hypothesis : Capitalist processes and the behaviour of the economy", in : C. Kindleberger et J.-P. Laffargue (eds.), 1982, Financial Crises. Theory, History, and Policy, Cambridge University Press ; M. H. Miller, 1986, "Financial Innovation: The Last Twenty Years and the Next", in The Journal of Financial and Quantitative Analysis, Vol. 21, No. 4, pp. 459- 471.

5. Cf. par exemple: The International Handbook on Social Innovation Collective Action, Social Learning and Transdisciplinary Research, Édité par F. Moulaert, D. MacCallum, A. Mehmood, et A. Hamdouch. Edward Elgar Pub., 2013.

6. Cf. par exemple OCDE, Measuring Innovation in Education, 2014. De façon différente, pour illustrer, cf. Le Conseil national de l'innovation pour la réussite éducative, installé le 19 avril 2013. Cf. aussi : M. Guiney, 2014, «L'innovation pédagogique par le bas ? PISA et la profanation de l'école-sanctuaire par la mondialisation dans l'enseignement littéraire ", in Revue de Métaphysique et de Morale, $\mathrm{n}^{\circ}$ 3/2014 (numéro spécial sur L'innovation).

7. Cf. par exemple: A. Oural, Rapport sur l'innovation territoriale du 24 avril 2015. L'innovation au pouvoir! Pour une action publique réinventée au service des Territoires (http://www.action-publique.gouv.fr/files/ files/PDF/2015_rapport_innovation_territoriale.pdf)

8. Cf. par exemple : Doner, R. F. (ed.), 2010, Explaining Institutional Innovation. Case Studies from Latin America and East Asia, Social Science Research Council (USA).

9. Cf. par exemple, La Fondation pour l'Innovation Politique qui publie annuellement un ouvrage sur l'innovation politique au cours de l'année passée (dernier paru : Innovation politique 2014, D. Reynié ed., P.U.F.).

10. S. Goyard-Fabre, 2014, «L'innovation dans le champ juridique in Revue de Métaphysique et de Morale, op. cit.

11. P. Capelle-Dumont, 2014, "L'innovation théologique », in Ibid.

12. Soulignons-le : nous faisons allusion ici à des discours et à des actions publiques qui se situent en dehors de la sphère économique et de l'innovation techno-économique.

13. L'innovation financière est très importante. Ne serait-ce que par son rôle dans la crise de 2008. Mais même si elle s'en distingue, on est à la périphérie immédiate de l'économie.

14. G. Mulgan et L. Pulford, 2010, Study on Social Innovation, European Union.

15. Revue de Métaphysique et de Morale, op. cit.

16. Tel est le point de vue développé par J. Schumpeter en 1911.

17. Une injonction est paradoxale dans la mesure où elle contient en elle-même sa propre contradiction : Innovez ! ressemble à l'injonction : Soyez spontanés !. Là où, au départ au moins (l'entrepreneur schumpétérien), l'innovation est un acte qui exprime une conviction, une inspiration ou un élan, l'injonction à innover engage les acteurs dans des activités programmées 
voire encadrées qui semblent n'avoir rien à voir avec un élan personnel reposant sur la conviction d'avoir une bonne idée. Mais si l'on considère que l'on peut innover sans inspiration particulière et à partir d'opérations programmées et structurées a priori, l'injonction à innover ne paraîtra pas paradoxale...

18. M. Hubert, F. Chateauraynaud et J.-M. Fourniau, «Les chercheurs et la programmation de la recherche: du discours stratégique à la construction de sens » in Quaderni, n 77(1), 2012, pp. 85-96.

19. L'entrepreneur schumpeterien est caractérisé par une volonté de réalisation de soi dans 1'acte même d'innover et de diriger une équipe. Mais aujourd'hui c'est différent : la valorisation de chacun pourvu qu'il innove ne renvoie pas à un motif ou une pulsion de réalisation personnelle. Elle renvoie à une évaluation sociale de l'acte et de la performance (plus ou au moins autant que le résultat de l'innovation). C'est plus proche de la reconnaissance sociale que de l'accomplissement personnel. Il peut cependant y avoir convergence, ce qui ne retire rien aux différences.

20. Rappelons que $1^{\prime}$ entrepreneur au sens de Schumpeter, qui est la personnification de la fonction d'innovation, selon un procédé méthodologique caractéristique de l'école de pensée économique autrichienne, est d'une autre nature que $l^{\text {'entrepreneur }}$ de J.-B. Say ou que l'entrepreneur "Marshallien".

21. Le linguistic turn (expression attribuée au philosophe R. Rorty) peut être défini par l'application du principe que le langage est la médiation nécessaire pour penser l'expérience et son rapport à la « réalité ». 22. La responsable politique (N. Kosciusco-Morizet) qui, récemment, déclarait qu' "Une ville innovante n'est pas une ville qui parle d'innovations mais une ville qui innove » oublie (ou feint d'oublier) que la question se pose de savoir d'abord ce que c'est qu'innover. Cette déclaration suppose que le mot innovation est associé à un signifié et à un référent uniques alors que la diffusion et l'usage du mot dans des contextes et des domaines variés ne peuvent qu'en faire douter. La déclaration dont nous parlons n'était cependant pas dénuée d'intérêt puisqu'elle visait à observer que «faire de l'innovation un slogan n'en fait pas une réalité».

23. L. Sfez, Technique et idéologie, Seuil, 2002.

24. B. Godin cite, à juste titre, R. Koselleck et J. Pocock, mais pas, dans cet article, pour la dimension socio-historique de la sémantique; pour 1'usage qu 'ils font du mot innovation.

25. B. Müller, 1993, Compte rendu de R. Koselleck : « Le futur passé, contribution à la sémantique des temps historiques », Éditions de l'École des Hautes Études en Sciences Sociales, 1990, Revue Suisse d'Histoire, vol.43.

26. L'existence de capacités entrepreneuriales et celle d'un système bancaire créateur de monnaie.

27. Pour une synthèse des risques et des dérives de l'innovation technologique $\mathrm{cf}$. D. Blondel, La valeur équivoque des innovations stimulées par le progrès des technologies, Académie des Technologies, janvier 2013.

28. Nous ne développons pas volontairement ce point ici. La littérature abondante est notamment dominée par les contributions de H. Marcuse, J. Ellul, L. Sfez, J. Habermas...

29. Cf. par exemple : R. Florida, "Technology Policy for a Global Economy”, Issues in Science and Technology, printemps 1995.

30. Nous renvoyons le lecteur à l'ouvrage d'Y. Bonny, Sociologie du temps présents. Modernité avancée ou postmodernité ? Armand Colin, 2004.

31. Cf. B. Godin, L'innovation : un slogan, rien qu'un slogan (GRANDLYON, Direction de la Prospective et du dialogue public).

32. Pour illustrer, cf. H. Fisher, « La société de 1'innovation. Les laboratoires du futur », publié dans 
Le Devoir du 22 janvier 2002.

33. P. Thibaud, 1985, "La société d'innovation », in Esprit, janvier 1985.

34. B. Paulré et alii, 1999, Le capitalisme cognitif comme sortie de la crise du capitalisme industriel. Un programme de recherche, Université Paris 1, ISYSMATISSE. Présenté au Forum de la régulation.

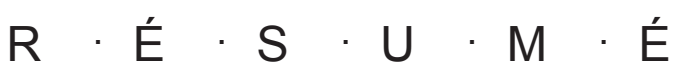

Si l'innovation technologique a monopolisé l'attention tout au long du $\mathrm{XX}^{\mathrm{e}}$ siècle, nous sommes aujourd'hui confrontés à une situation originale caractérisable par trois traits : 1) il est de plus en plus question d'innovation en dehors du champ économique pour désigner des activités créatrices de nouveauté dont les modalités et les contenus sont sensiblement différents de ceux de l'innovation techno-économique ; 2) Il existe de multiples injonctions à innover, et 3) l'innovation est souvent l'objet d'une valorisation en soi.

Ces trois traits seront argumentés successivement avant que l'on suggère quelques pistes d'interprétation de cette nouvelle situation. Pour cela, nous distinguerons la réflexion conceptuelle de celles relatives à la signification historique de l'évolution en cours : S'agit-il de la manifestation d'une emprise accrue du néo-libéralisme sur les sociétés développées contemporaines, de la manifestation de ce que, génériquement, nous pouvons appeler l'après-modernité, ou encore des signes de l'émergence d'une société d'innovation?

\section{Abtract}

Innovative trends in contemporary societies

Throughout the twentieth century technological innovation has monopolized the attention, but we are now facing a new situation characterized by three features : 1) we are concerned more and more with innovation outside of the economic field, that is to say creative activities of novelty, of which the terms and contents are appreciably different from those of the technoeconomic innovation, 2) there are many injunctions to innovate and 3) innovation is often the subject of a valuation in itself.

These three features will be argued successively be- 
fore one suggests some ways of interpreting this new situation. For that, we will distinguish the conceptual thinking from those relating to the historical significance of the evolution in progress: Is it the demonstration of an increased influence of the neoliberalism on the contemporary developed companies ? Or the demonstration of what, generically, we can call aftermodernity, or signs of the emergence of an innovation society? 